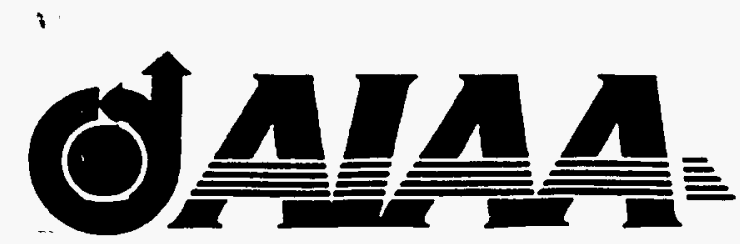

\title{
AIAA 99-0756 \\ Large Eddy Simulation of Aircraft Wake Vortices in a Homogeneous Atmospheric Turbulence: Vortex Decay and Descent
}

Jongil Han, Yuh-Lang Lin, S. Pal Arya North Carolina State University

Raleigh, North Carolina

Fred H. Proctor

NASA Langley Research Center

Hampton, Virginia

\section{7th AIAA Aerospace Sciences Meeting and Exhibit January 11-14, 1999 / Reno, NV}




\title{
LARGE EDDY SIMULATION OF AIRCRAFT WAKE VORTICES IN A HOMOGENEOUS ATMOSPHERIC TURBULENCE: VORTEX DECAY AND DESCENT
}

\author{
Jongil $\operatorname{Han}^{*}$, Yuh-Lang $\operatorname{Lin}^{\dagger}$, S. Pal Arya ${ }^{\ddagger}$ \\ Department of Marine, Earth and Atmospheric Sciences \\ North Carolina State University \\ Raleigh, NC 27695-8208 \\ Fred H. Proctor ${ }^{\S}$ \\ NASA Langley Research Center \\ Flight Dynamics and Control Division \\ Hampton, VA 23681-1001
}

\begin{abstract}
$\underline{\text { Abstract }}$
The effects of ambient turbulence on decay and descent of aircraft wake vortices are studied using a validated, three-dimensional, large-eddy simulation model. Numerical simulations are performed in order to isolate the effect of ambient turbulence on the wake vortex decay rate within a neutrally-stratified atmosphere. Simulations are conducted for a range of turbulence intensities, by injecting wake vortex pairs into an approximately homogeneous and isotropic turbulence field. The decay rate of the vortex circulation increases clearly with increasing ambient turbulence level, which is consistent with field observations.

Based on the results from the numerical simulations, simple decay models are proposed as functions of dimensionless ambient turbulence intensity $(\eta)$ and dimensionless time $(T)$ for the circulation averaged over a range of radial distances. With good agreement with the numerical results, a Gaussian type of vortex decay model is proposed for weak turbulence, while an exponential type of vortex decay model can be applied for strong turbulence. A relationship for the vortex descent based on above vortex decay model is also proposed. Although the proposed models are based on simulations assuming neutral stratification, the model predictions are compared to Lidar vortex measurements observed during stable, neutral, and unstable atmospheric conditions. In the neutral and unstable atmosphere, the model predictions appear to be in reasonable agreement with the observational data, while in the stably-stratified atmosphere, they largely underestimate the observed circulation decay with consistent overestimation of the observed vortex descent. The underestimation of vortex decay during stably-stratified conditions suggests that stratification has an important influence on vortex decay when ambient levels of turbulence are weak.
\end{abstract}

\section{Introduction}

For the purpose of increasing airport capacity, a system is being developed under the National Aeronautic and Space Administration's (NASA) Terminal Area Productivity (TAP) program that will con-

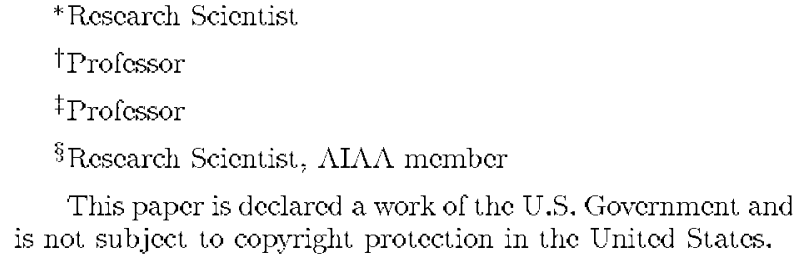

This paper is declared a work of the U.S. Government and is not subject to copyright protection in the United Statcs.

trol aircraft spacing within the narrow approach corridors of airports. The system, called the Aircraft Vortex Spacing System ${ }^{1,2}$ (AVOSS), will determine safe operating spacing between arriving and departing aircraft as based on the observed or predicted weather conditions. This system will provide a safe reduction in separation of aircraft compared to the now-existing flight rules, which are based on aircraft weight categories. In order to develop this system, research is being focused on understanding how aircraft wake vortices interact with the atmosphere. Previous studies indicate that transport and decay 
of wake vortices are strongly affected by ambient atmospheric parameters such as wind shear, stratification, and turbulence, as well as by proximity of aircraft to the ground. ${ }^{3}$ In this study we focus on the effects of three-dimensional ambient atmospheric turbulence on vortex decay and descent using a validated large eddy simulation (LES) model.

The mechanisms and causes for the decay of the wake vortices are somewhat controversial. ${ }^{4}$ Some researchers believe that vortices generally do not decay until three-dimensional instabilities such as Crow instability ${ }^{5}$ lead to their sudden destruction. ${ }^{6}$ However, this view is held in spite of the overwhelming observational evidence that vortices decay at different rates depending upon the ambient meteorology, ${ }^{3}$ and atmospheric turbulence plays a key role. ${ }^{7,8}$ Using a second-order turbulence closure model, Bilanin et al. ${ }^{9}$ shows that the rate of decay of a vortex pair increases with increasing background dissipation rate. Recent analyses of field observation data by Sarpkaya ${ }^{10}$ clearly show that vortex circulation decays much faster in the presence of strong turbulence than in weak turbulence. The analyses of LES data $^{3,11,12}$ also reveal that three-dimensional atmospheric turbulence enhances circulation decay. On the other hand, the descent speed of a vortex pair due to mutual induction decreases with increasing circulation decay as long as the separation distance of a vortex pair remains constant. From data obtained in the water tank experiments, Sarpkaya and Daly ${ }^{13}$ find that the descent speed of vortices decreases with increasing dissipation rate of the ambient turbulence (i.e., increasing ambient turbulence level), which in turn implies that stronger turbulence yields larger reduction in circulation. Stable stratification can also hasten vortex decay through baroclinic generation of counter-sign vorticity along the vortex oval's periphery. ${ }^{3,14,15}$ Since most of field observation data are subjected to the influence of both turbulence and stratification, it is difficult to distinguish their effects on circulation decay and vortex descent. In the present study, we consider only a neutrally stratified atmosphere in order to isolate atmospheric turbulence effects on vortex decay. Investigations of wake vortex decay in a non-neutral environment are ongoing and will be reported in a future paper.

The enhancement of vortex decay due to ambient turbulence has been an important factor in wake-prediction models. ${ }^{7,8}$ Donaldson and Bilanin ${ }^{7}$ proposed a formula describing the relation between atmospheric turbulence and vortex circulation:

$$
\frac{d \Gamma}{d t}=-0.82 \frac{q \Gamma}{b_{0}},
$$

where $\Gamma$ is circulation, $q=\left(\overline{u^{2}}+\overline{v^{2}}+\overline{w^{2}}\right)^{1 / 2}$ is the turbulent velocity scale, and $b_{0}$ initial separation distance of a vortex pair. Greene ${ }^{8}$ incorporated $E q .(1)$ into his analytic model for the contribution of atmospheric turbulence effects on wake vortex motion and decay. Equation (1) implies that vortex circulation decays exponentially and depends only on ambient turbulence, represented by $q$ which is related to the turbulence kinetic energy $\left(T K E=q^{2} / 2\right)$. The $T K E$ or $q$ is primarily determined by large energycontaining eddies. According to $E q$.(1), then, large scale eddies are related to the decay of vortex circulation. Although, large energy-containing eddies can lead to a large deformation and transport of vortices as well as to the development of the large scale Crow instability, ${ }^{3,16,17,18}$ it is the small scale (order of $b_{0}$ ) turbulent eddies that are directly associated with the decay of vortex circulation. These smaller scales of turbulent eddies are likely to be more homogeneous and isotropic and mostly lie within the Kolmogorov inertial subrange which is characterized only by turbulent kinetic energy dissipation rate $(\epsilon){ }^{19}$ Therefore, one can use $\epsilon$ rather than $q$ as the turbulence parameter for controlling the vortex decay. The two are related as $\epsilon=q^{3} / l_{\epsilon}$, where $l_{\epsilon}$ is a characteristic large-eddy length scale, called the dissipation length.

In fact, $\epsilon$ has been commonly used in the literature ${ }^{13,17,18,20,21}$ as an appropriate parameter to investigate the effects of ambient turbulence on the lifespan of wake vortices. The strength of turbulence is often represented by the dimensionless turbulence intensity $\eta$, defined as

$$
\eta=\frac{\left(\epsilon b_{0}\right)^{1 / 3}}{V_{0}}
$$

where

$$
V_{0}=\frac{\Gamma_{\infty}}{2 \pi b_{0}} .
$$

Here, $\Gamma_{\infty}$ represents circulation at very large radial distance. Note that $\eta$ represents the ratio of the characteristic turbulent velocity scale at the scale of the vortex separation distance $\left(\left(\epsilon b_{0}\right)^{1 / 3}\right)$ to the speed of descent of the vortex pair by mutual induction $\left(V_{0}\right)$.

Our studies are focused on the far-field, post rollup stage of the vortex pair, in which each vortex possesses a well-developed structure. For later discussion, we define nondimensional parameters for time 
$(t)$, descent distance of the vortices $(h)$, and radial distance $(r)$ as

$$
T=\frac{V_{0} t}{b_{0}}, \quad H=\frac{h}{b_{0}}, \text { and } R=\frac{r}{b_{0}} .
$$

In Section 2, we describe the LES model and the modifications required for initial conditions. In Section 3 , we present the results from systematic numerical experiments in terms of nondimensional turbulence intensity and also propose new vortex models for vortex decay and descent, based on the LES results. In Section 4, we apply the proposed models to available observation data. Finally, in Section 5, we summarize our results and draw some conclusions.

\section{The model and initial conditions}

\subsection{The model}

The numerical model used in the present study is a three-dimensional, nonlinear, compressible, nonhydrostatic LES model, called the Terminal Area Simulation System ${ }^{22}$ (TASS), which has been adopted for simulation of interaction of wake vortices with the atmosphere. ${ }^{23,24,25}$ Recently, this code has been used to study the development of the Crow instability in an artificially generated homogeneous atmospheric turbulence. ${ }^{18}$ The agreement between the calculated and measured maximum instability wavelengths and vortex lifespans provided a good validation of the present TASS LES code. The model equations are basically identical to those used in Han et al. ${ }^{18}$ except for the modified subgrid model which accounts for the flow rotation effects.

Turbulence is strongly affected by the rotation of the swirling flow of vortex. According to Rayleigh's well-known stability criterion, ${ }^{26}$ perturbations are suppressed in an axisymmetric vortex if the circulation is increasing with radial distance, but perturbations would become unstable if the circulation is decreasing with radial distance. Since the circulation increases rapidly with radial distance in the vortex core region, any pre-existing turbulence is suppressed there and the vortex core ${ }^{\text {I }}$ expands very little with time. ${ }^{15}$ In the present numerical model, a scheme for the subgrid eddy viscosity which takes account of the flow rotation effects is used to avoid unrealistic core growth.

\footnotetext{
T The vortex core is defined as the radius of maximum tangential velocity.
}

For subgrid turbulence, TASS currently uses a conventional Smagorinsky model ${ }^{27}$ with modifications for stratification effects as:

$$
\begin{aligned}
K= & \left(c_{s} \Delta\right)^{2}|D|\left(1-\alpha R i_{S}\right)^{0.5}, \\
& R i_{S}=\frac{N^{2}}{D^{2}}, \text { and } N^{2}=\frac{g}{\theta} \frac{\partial \theta}{\partial z}
\end{aligned}
$$

where $K$ is the subgrid eddy viscosity, $R i_{S}$ is the Richardson number due to stratification, $\Delta$ is the filter width, $D$ is the rate of deformation, $N$ is the Brunt-Väisäla frequency, $g$ is the gravity, $\theta$ is the potential temperature, and $c_{s}=0.16$ and $\alpha=3$ are constants. After initial sensitivity tests, we have realized that the Smagorinsky subgrid closure model overpredicts the decay rate in the vortex core region and causes unrealistic growth of the core size. Since vortices have been observed to maintain their core strength and size for a much longer time, ${ }^{10}$ it is necessary to incorporate the rotation effects into the Smagorinsky model. A simple modification of the eddy viscosity to include rotation effect analogous to the stratification effect has been developed. ${ }^{28}$

The modified subgrid eddy viscosity is given as

$$
K=\left(c_{s} \Delta\right)^{2}|D|\left(1-\alpha R i_{S}-\beta R i_{R}\right)^{0.5} .
$$

Here $R i_{R}$ is the rotational Richardson number, defined $\mathrm{as}^{28}$

$$
R i_{R}=\frac{\Omega^{2}}{D^{2}}+\frac{|\Omega|}{|D|}
$$

where $\Omega$ is the magnitude of three-dimensional vorticity. Equations (6) and (7) indicate that the eddy viscosity, $K$, can be effectively reduced within the core of vortices due to large $|\Omega| /|D|$. Since the above formula cannot discriminate between shear flow and flow with coherent rotation, a discriminator function is applied that turns the formula off (defaulting to the original Smagorinsky model) in the absence of coherent rotation. Details of this formula can be found in Ref. 28.

Periodic boundary conditions are imposed at all domain boundaries. Domain size used in our simulations is $\left(L_{x}, L_{y}, L_{z}\right)=\left(2.5 b_{0}, 5 b_{0}, 5 b_{0}\right)=(80 \Delta x$, $160 \Delta y, 160 \Delta z)$ where $b_{0}=32 m$, grid size $\Delta x=\Delta y$ $=\Delta z=1 \mathrm{~m}$, and $x, y$ and $z$ correspond to the axial, lateral and vertical directions of the vortex system and corresponding velocity components are $u, v$ and $w$, respectively. The smaller domain size in the axial direction $\left(2.5 b_{0}\right)$, which can save much computing time, suppresses the development of Crow instability of which theoretical maximum wavelength is about 

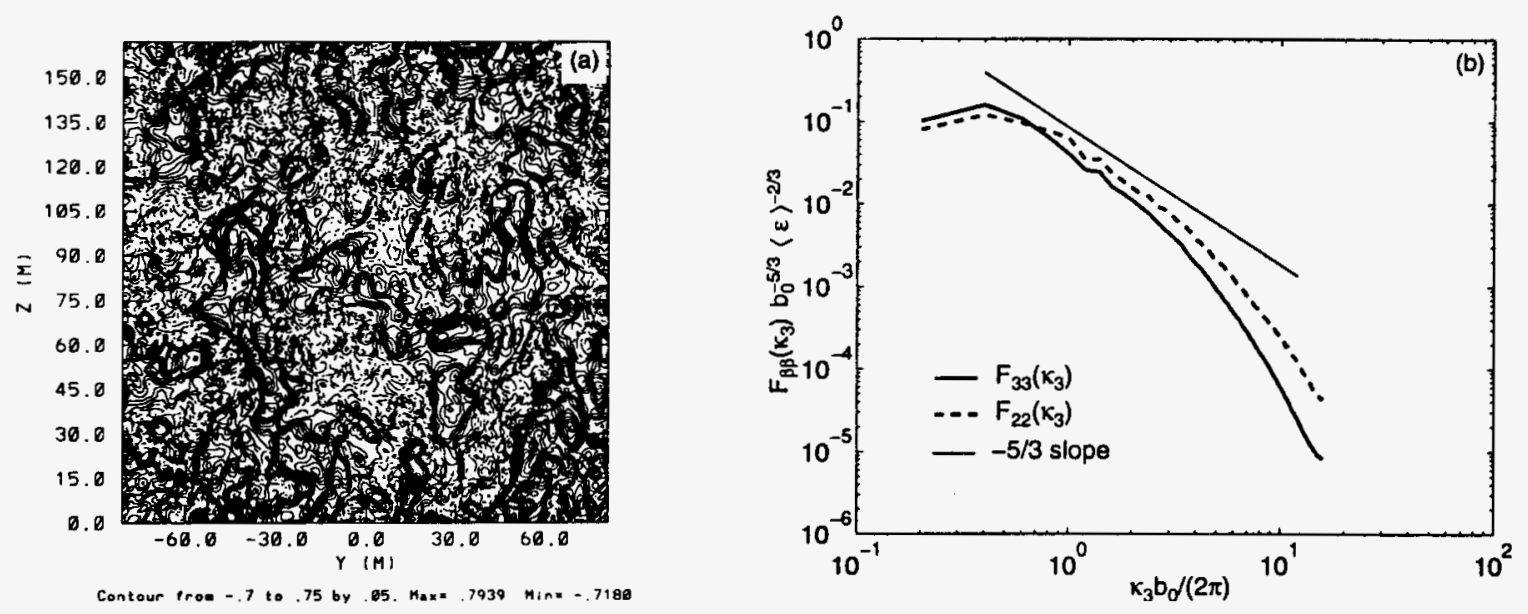

Figure 1: (a) Vertical velocity field and (b) its one-dimensional energy spectrum in a steady state turbulence before vortex injection.

$8.6 b_{0},{ }^{5}$ and thus statistically homogeneous decay behavior is anticipated along the axial direction. The domain size of $5 b_{0}$ in lateral and vertical directions is sufficiently large to minimize boundary influences. Due to limited computing resources and in order to allow the core to be resolved, the initial core size $\left(r_{c}=4 m\right)$ is somewhat larger than the typical value observed behind aircraft (about $0.05 B^{23}$ ). Ground effects are not included in the simulations, thus results are applicable only to out-of-ground-effect wake vortex behavior.

\section{$\underline{2.2}$ Initial conditions}

Since we want to study the effect of ambient turbulence on the vortex decay and descent, it is of crucial importance to obtain an initially homogeneous and isotropic turbulence field. Toward this purpose, the initial turbulence field is allowed to develop under an artificial external forcing at low wavenumbers using a three-dimensional Fast Fourier Transform, as described in Han et al. ${ }^{18}$

Figure 1 shows the vertical velocity field and its one-dimensional longitudinal and transverse spectrum with $-5 / 3$ slope of Kolmogorov's spectrum when the turbulent flow field reaches a statistically steady state in which the mean turbulence kinetic energy fluctuates in time around a constant value due to viscous dissipation against external forcing. The integral length scale is calculated as $L_{33} \approx 11.4 \mathrm{~m}$ and the large-eddy turnover time, defined as $t_{e}=$ $L_{33} /\left\langle w^{2}\right\rangle^{1 / 2}$, is estimated to be about $51.6 s$. Here subscript 3 denotes vertical direction and (> represents the domain average value. The isotropy parameter $I$, defined as $I_{1}=\left[\left\langle u^{2}\right\rangle /\left\langle w^{2}\right\rangle\right]^{1 / 2}$ or $I_{2}=$ $\left[\left\langle v^{2}\right\rangle /\left\langle w^{2}\right\rangle\right]^{1 / 2}$, fluctuates only a few percent around its expected value of one for isotropic turbulence. Therefore, our simulated turbulence is close to statistical isotropy. The TKE dissipation rate $\epsilon$ is estimated from the well known form of the spectrum in the inertial subrange and fitting the same to the simulated spectra in Fig. 1.

The initial vortex system is representative of the post roll-up, wake-vortex velocity field. A vortex model recently developed by Proctor ${ }^{3}$ is adopted in our experiments. This model is empirical as it is based on field observations of several wake vortices measured early in their evolution. Its tangential velocity, $V$, is represented as

$$
V(r)=\frac{\Gamma_{\infty}}{2 \pi r}\left(1-e^{-10(r / B)^{0.75}}\right),
$$

where $\Gamma_{\infty}$ is the circulation at $r \gg r_{c}\left(r_{c}\right.$ is the initial vortex core radius defined as radial distance of peak tangential velocity) and $B$ is the span of the generating aircraft. The values assumed for initial vortex separation and circulation are derived from aircraft weight $(W)$, wingspan $(B)$, air density $(\rho)$, and airspeed $\left(V_{a}\right)$ according to the conventional assumption of elliptically-loaded wing, i.e.,

$$
b_{0}=\frac{\pi B}{4} \text { and } \Gamma_{\infty}=\frac{4 W}{\pi B \rho V_{a}} .
$$


Note that the velocity field in $E q .(8)$ depends on the wingspan $B$ instead of the core radius $r_{c}$ which may not be easy to measure with a good accuracy; $E q .(8)$ is applied only at $r>r_{c}$. For $r<r_{c}$, the model is matched with the Lamb model, ${ }^{29}$ i.e.,

$$
\begin{aligned}
V(r)= & \frac{\Gamma_{\infty}}{2 \pi r} 1.4\left(1-e^{-10\left(r_{c} / B\right)^{0.75}}\right) \\
& \left(1-e^{-1.2527\left(r / r_{c}\right)^{2}}\right) .
\end{aligned}
$$

Normalized circulation, tangential velocity, and vorticity from the above model are shown in Fig. 2 as function of the normalized radial distance $R=r / b_{0}$, assuming $r_{c} / b_{0}=0.125$.

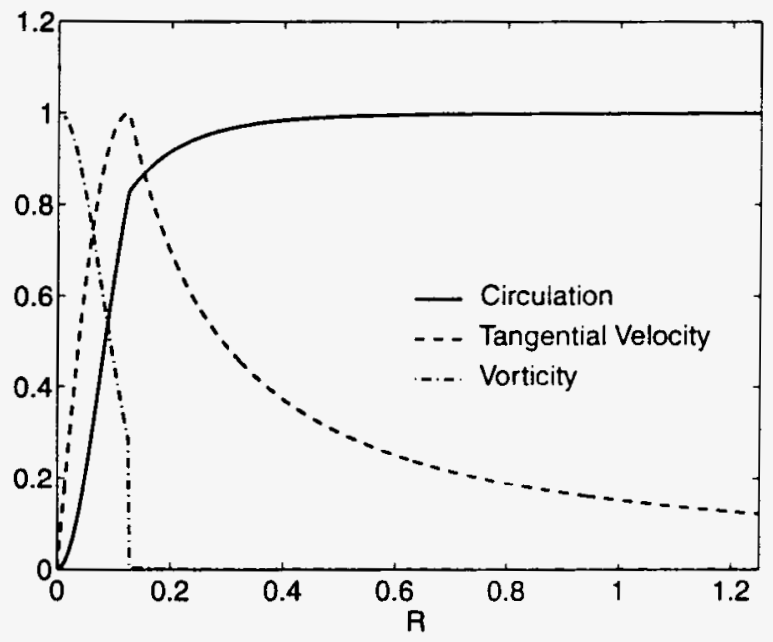

Figure 2: Initial circulation, tangential velocity, and vorticity for each vortex normalized by their maximum values.

A two-dimensional counter-rotating vortex pair prescribed by $E q .(8)$ and $E q .(10)$ is initialized uniformly along the axial direction at the time when the ambient turbulence reaches a steady state after integration. In order to maintain the vortices in the middle of the domain, the grid is allowed to translate downward at the speed of $90 \%$ of $V_{0}$. Nondimensional ambient turbulence strength, $\eta$, is obtained by varying the circulation rather than by varying $\epsilon$ to save computing time and thus all the following results are presented as nondimensional forms. Assuming that $\Gamma_{\infty}=400 \mathrm{~m}^{2} \mathrm{~s}^{-1}$ and $b_{0}=40 \mathrm{~m}$, which are typical for $D C-10$ aircraft, the range of typical values of $\eta$ in the lower atmosphere is about 0.01-0.5, with the maximum in the early afternoon and the minimum after midnight ${ }^{3}$. Simulations are conducted for six values of $\eta$, which are within this typical range. These cases are divided into three turbulence strength groups: $\eta=0.0302,0.0702$ for weak turbulence, $\eta=0.1006,0.1509$ for moderate turbulence, and $\eta=0.3018,0.5031$ for strong turbulence, where "weak" represents the strength of typical boundary layer turbulence during stable night-time conditions and "strong" represents typical strength during sunny daytime conditions. In order to isolate atmospheric turbulence effect on vortex decay, the numerical simulations presented in this paper assume neutral stratification.

\section{Numerical results}

External forcing for maintaining ambient turbulence strength is deactivated to save computing time when the initial vortices are injected, but the difference in the result for activating or deactivating the forcing has been found to be negligible (not shown). This confirms the arguments by Corjon et al..$^{30}$ that the time scale of the ambient turbulence compared to that of the vortex is sufficiently large to obtain the main characteristics of the effects of ambient turbulence on the wake vortices.

During simulations, the vortices descend smoothly without any development of large scale instabilities such as Crow instability, which is not allowed due to the small domain size used in the axial direction.

The circulation at any radial distance $r$ can be easily determined from the area integral of the axial vorticity $(\zeta)$ over a region defined by the radial distance, i.e.,

$$
\Gamma_{r}=\iint \zeta d A .
$$

Obtained at each radial distance are a total 160 circulation values for a pair of vortices in $80 y-z$ planes from which we have calculated averages and standard deviations. As shown in Table 1, the circulation fluctuations due to ambient turbulence increase with increasing turbulence level. The standard deviations appear to be small for most of turbulence levels (less than 10\%) at least at the end of the simulation time, except for the strongest turbulence strength of $\eta=0.5031$ for which the values are more than $20 \%$. In the following, we consider only the average of circulations to deduce an appropriate model for vortex decay and descent.

\subsection{Circulation decay}




\begin{tabular}{|c|c|r|r|r|}
\hline \hline$\eta$ & $T$ & $3 r_{c}$ & $4 r_{c}$ & $5 r_{c}$ \\
\hline 0.0302 & 7 & 2.8 & 2.0 & 2.1 \\
0.0702 & 6 & 3.8 & 4.1 & 4.3 \\
0.1006 & 6 & 3.5 & 4.5 & 4.6 \\
0.1509 & 6 & 6.6 & 9.0 & 9.9 \\
0.3018 & 3 & 10.1 & 9.6 & 8.5 \\
0.5031 & 3 & 31.4 & 28.2 & 23.1 \\
\hline \hline
\end{tabular}

Table 1: Standard deviation of the circulation relative to its mean value (\%) at varying radial distances and dimensionless turbulence strength $\eta$ at the final dimensionless simulation time $(T)$.

Figure 3 shows the decay of circulation with time for varying radial distances as well as varying ambient turbulence levels. As shown in Fig. 3, the decay rate of the circulation increases clearly with increasing ambient turbulence level and appear to decrease with increasing radial distance, which is consistent with field observations. ${ }^{10}$

In order to investigate the vortex decay behavior in more detail for varying radial distances, the circulation evolution with a best-fitted function for each radial distance is plotted in Fig. 4 for relatively weak turbulence $(\eta=0.0702)$, in Fig. 5 for relatively moderate turbulence $(\eta=0.1509)$, and in Fig. 6 for strong turbulence $(\eta=0.5031)$. As evidenced in Figs. 4-6, the decay of the circulation appears to follow a Gaussian function, $e^{-a T^{2}}$, for weak and moderate turbulence (hereafter model G), while for strong turbulence, it appears to follow an exponential function, $e^{-b T}$, at smaller radial distances (hereafter model $E$ ) but a Gaussian function at larger radial distances, where $a$ and $b$ would be functions of $\eta$ and radial distance. Although partly shown in Figs. 4-6, the circulation at radial distances larger than $0.6 b_{0}$ appears to follow the Gaussian decay for all levels of turbulence. An exponential decay formula, $E q .(1)$, was first proposed by Donaldson and Bilanin, ${ }^{7}$ with $q$ rather than $\epsilon$ as the turbulence parameter controlling the vortex decay. The Gaussian type of the decay (model $G$ ) replicates the much slower decay during early evolution of the vortices. This slow decay at earlier time has also been reported in Corjon and Darracq's ${ }^{31}$ LES results, in which the circulation begins to decay after a period of about $10 \mathrm{~s}$. Based on our LES results described above, two types of models (i.e., model $\mathrm{E}$ and model G) are proposed for vortex decay and their formulations are given in Appendix A.

Previous investigators have quantified the strength of a vortex in terms of a parameter called average circulation, which is defined as ${ }^{23,32}$

$$
\bar{\Gamma}_{r_{1}, r_{2}}=\frac{\int_{r_{1}}^{r_{2}} \Gamma d r}{\int_{r_{1}}^{r_{2}} d r}
$$

where $r_{1}$ and $r_{2}$ are the radial distances of the averaging interval. This parameter is desirable, since it relates to the rolling moment of an encountering aircraft and provides a more stable measurement than the local circulation. ${ }^{23,32}$ In particular, the average circulation within $15 \mathrm{~m}$ from the vortex center has been considered to be closely related to the hazard. ${ }^{33,34}$ In the present study, we compute the average circulation from $r=0.4 b_{0}$ to $r=0.6 b_{0}$. The lower limit is large enough in order to avoid any artificial contamination near the core. The upper limit is small enough to minimize the effect of the accompanying vortex.

Since $\Gamma$ is a function of $R$, it is difficult to obtain decay formula for the average circulation directly from $E q .(A 14)$ and $E q .(A 19)$ based on the definition of the average circulation, i.e., $E q .(12)$. Instead, for the decay of the average circulation, $E q .(A 14)$ and $E q .(A 19)$ are approximated as

$$
\left.\frac{d \bar{\Gamma}}{d T}\right|_{\text {turbulence }}=-c_{1} \frac{\eta}{\bar{R}^{2}} \bar{\Gamma} \quad \text { for model } \mathrm{E}
$$

and

$$
\left.\frac{d \bar{\Gamma}}{d T}\right|_{\text {turbulence }}=-c_{2} \frac{\eta^{2} T}{\bar{R}^{2}} \bar{\Gamma} \quad \text { for model } \mathrm{G},
$$

where $c_{1}$ and $c_{2}$ are empirical constant and $\bar{R}$ is a value at the middle point of an average range of radial distances (e.g., $\bar{R}$ becomes 0.5 for the average range of $0.4 b_{0}-0.6 b_{0}$ used in the present study). Furthermore, integrations of $E q .(13)$ and $E q .(14)$ with respect to dimensionless timell (assuming ambient turbulence is only the process affecting vortex decay) yields

$$
\bar{\Gamma}=\bar{\Gamma}_{0} e^{-\left(c_{1} \eta / \bar{R}^{2}\right) T} \quad \text { for model } \mathrm{E}
$$

and

$$
\bar{\Gamma}=\bar{\Gamma}_{0} e^{-\left(c_{2} \eta^{2} / \bar{R}^{2}\right) T^{2}} \quad \text { for model G. }
$$

As can be seen from Fig. 7, the model predictions of average circulation agree much better with the LES data than those of local circulations, showing

"It is assumed that $\eta$ does not change with time. 

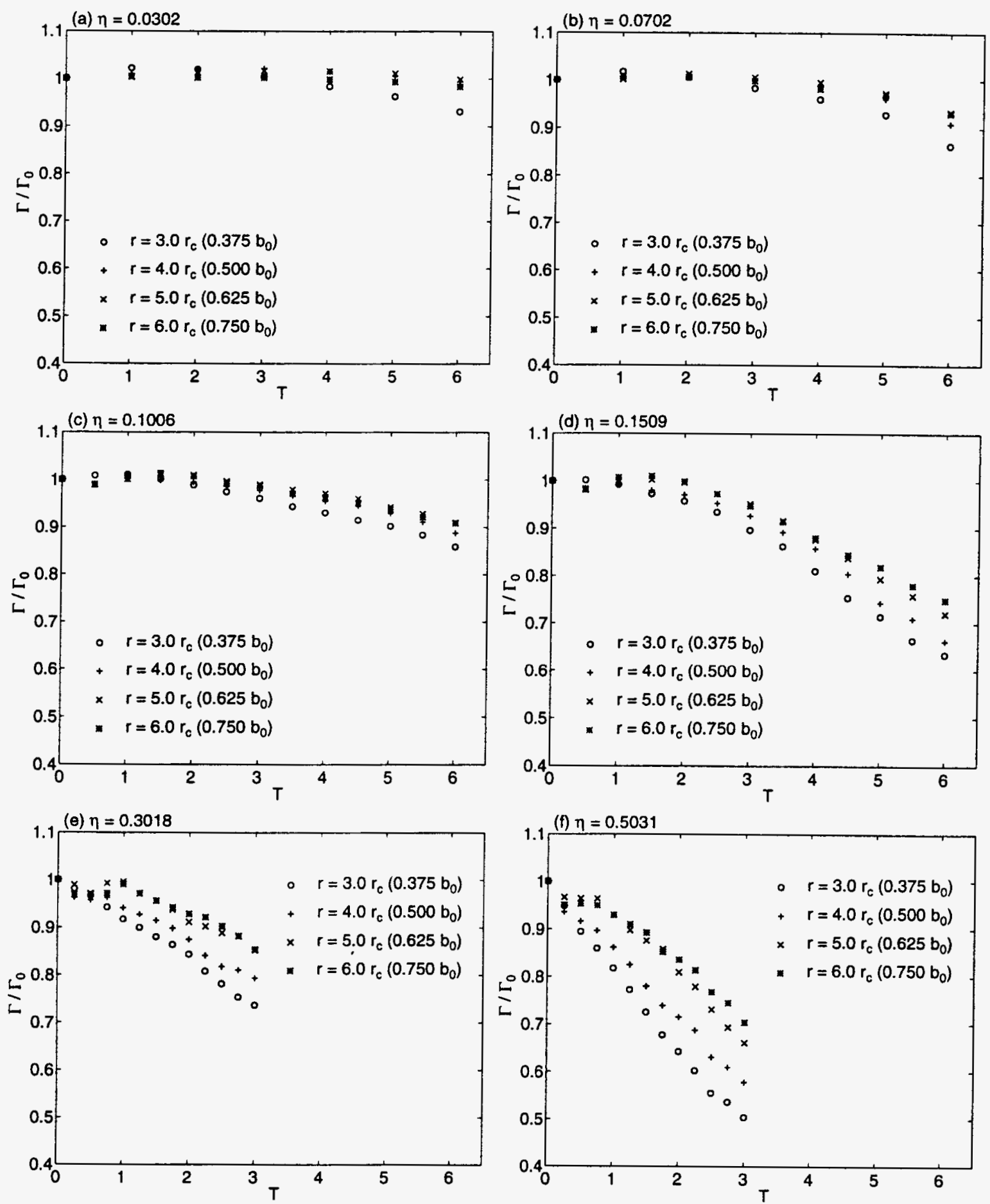

Figure 3: Decay of circulation with time for varying radial distances and ambient turbulence strengths.

7

American Institute of Aeronautics and Astronautics 

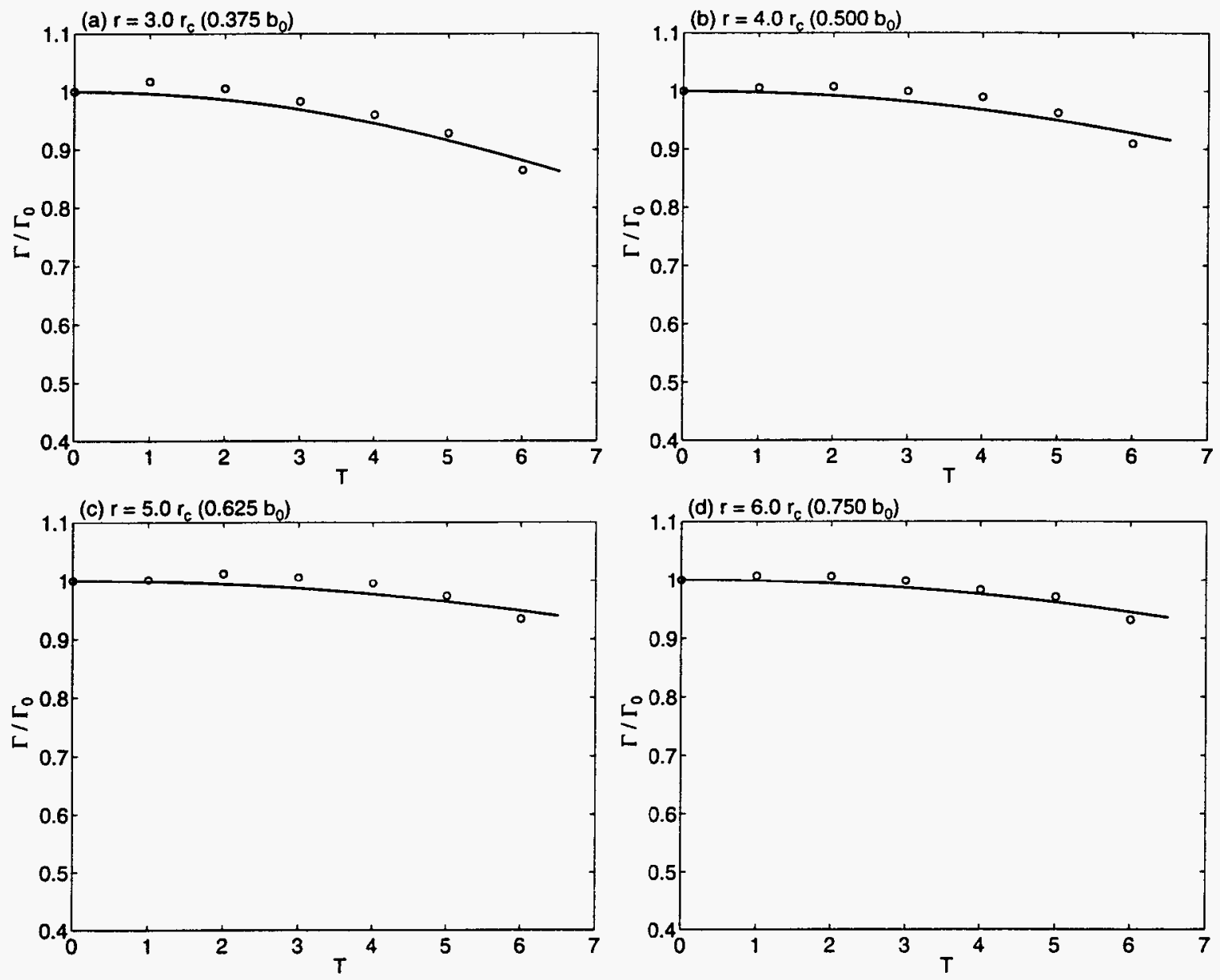

Figure 4: Circulation decay and best-fitted functions at four different radial distances for $\eta=0.0702$ : solid line indicates $e^{-a T^{2}}$, where $a$ is functions of $\eta$ and radial distance. 

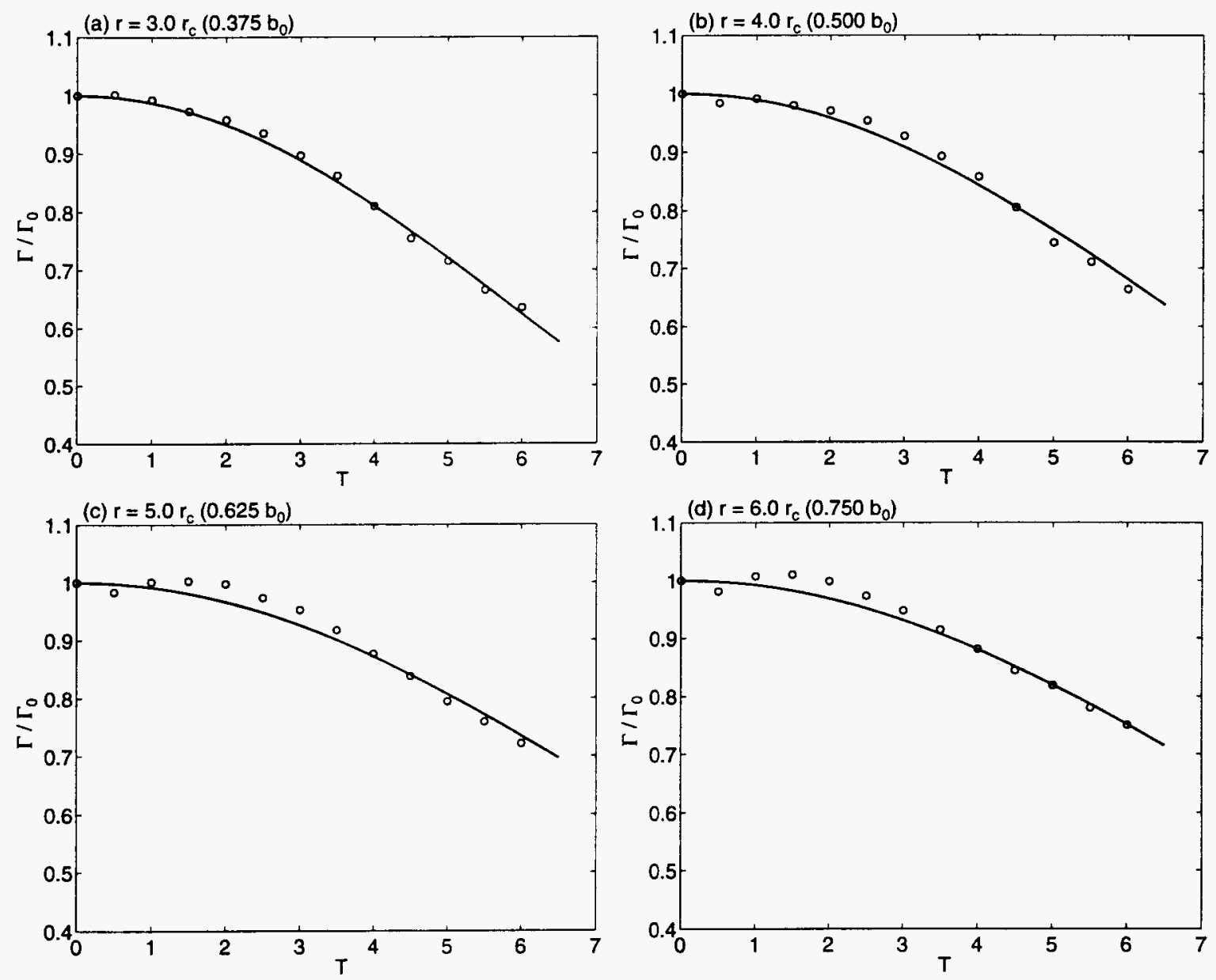

Figure 5: Same as Fig. 4 but for $\eta=0.1509$. 

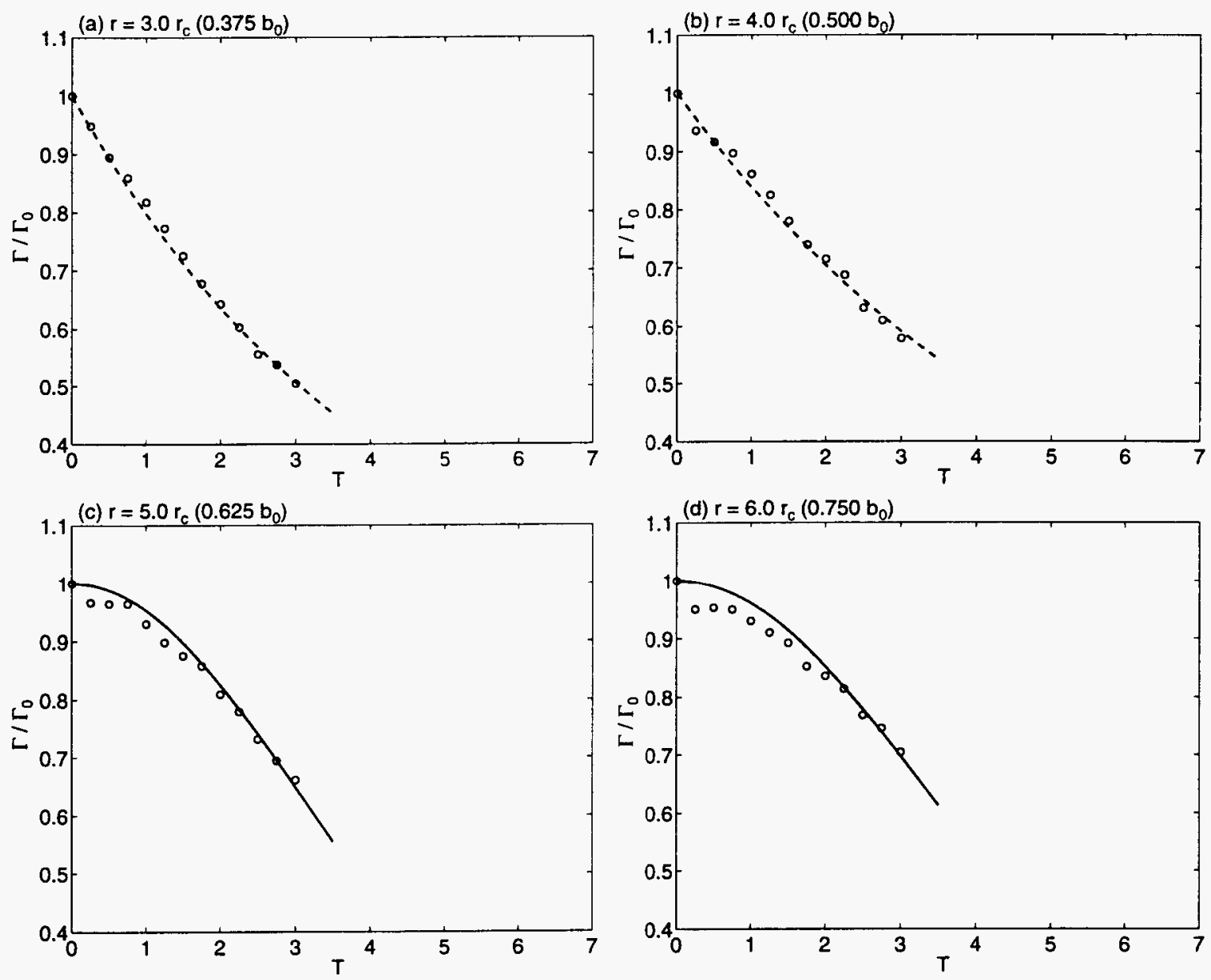

Figure 6: Same as Fig. 4 but for $\eta=0.5031$ : dashed line indicates $e^{-b T}$, where $b$ is functions of $\eta$ and radial distance. 

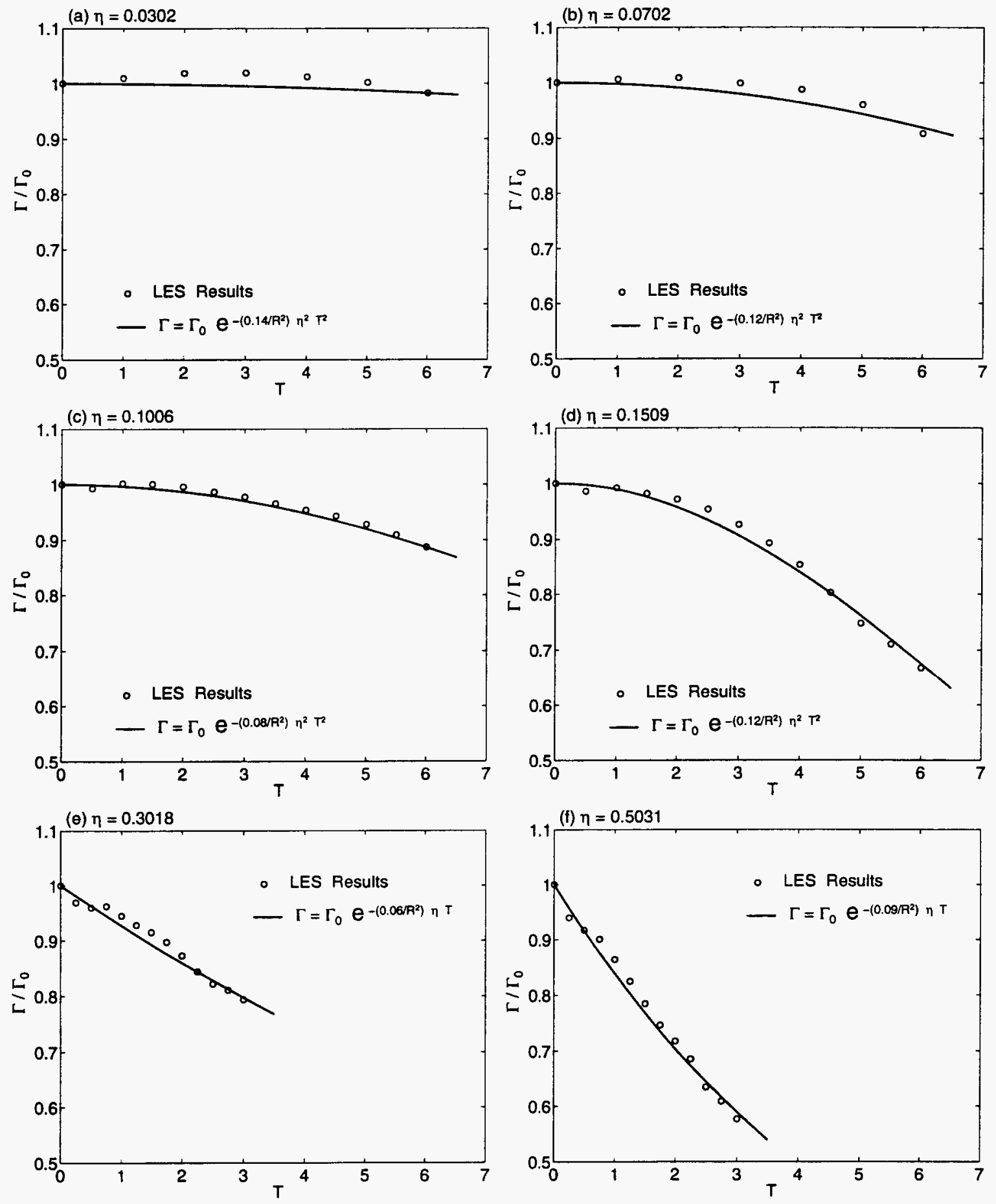

Figure 7: Evolution of the circulation averaged from $0.4 b_{0}$ to $0.6 b_{0}$ and corresponding model prediction with the coefficient adjusted for varying $\eta$. In figure, $\Gamma, \Gamma_{0}$, and $R$ represent $\bar{\Gamma}, \bar{\Gamma}_{0}$, and $\bar{R}$ in the text, respectively. 
a Gaussian decay for weak and moderate turbulence but an exponential decay for strong turbulence. The model coefficients do not appear to much fluctuate with varying $\eta$ in spite of the highly irregular nature of turbulence. In order to obtain a representative model coefficient, more experiments have been performed for different $\eta$ values and for different initial turbulence fields. The resulting coefficients (Fig. 8) fluctuate with varying $\eta$ within the ranges of $c_{1}=0.06-0.09$ and $c_{2}=0.08-0.20$, respectively. The mean values of the coefficients, which can be considered as representative coefficients, are 0.08 for model $E$ and 0.13 for model $G$, respectively.

Using the mean values of the coefficients, the model predictions of the average circulations for various ambient turbulence levels are plotted in Fig. 9. The vortex lifespans in Fig. 9, defined as the time at which linking of a vortex pair occurs, are obtained from a model recently developed by Sarpkaya. ${ }^{10}$ This model has been shown to agree well with data from water tank experiments ${ }^{13}$ and LES predictions. ${ }^{18}$ Figure 9 indicates that until a linking of the vortices occurs the circulation is reduced by only about $2 \%$ of its initial value for weak ambient turbulence $(\eta=0.03)$, while it may be reduced by as much as $20 \%$ for strong ambient turbulence $(\eta=0.50)$.

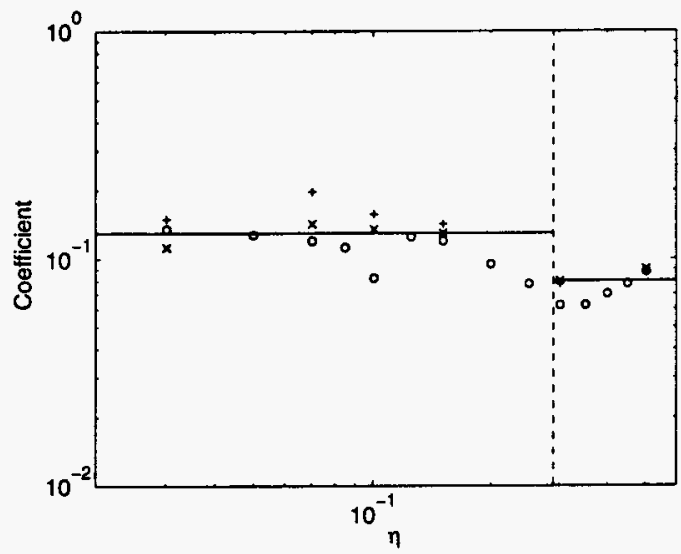

Figure 8: Model coefficients with varying $\eta$ for the circulation decay in $E q .(13)$ and $E q .(14)$ as obtained from numerical simulations. Different symbols represent the results from different initial turbulence fields. Solid lines denote the average of the coefficients for each model distinguished by dashed line.

\subsection{Vortex descent}

The descent of an ideal vortex pair, of which vor-

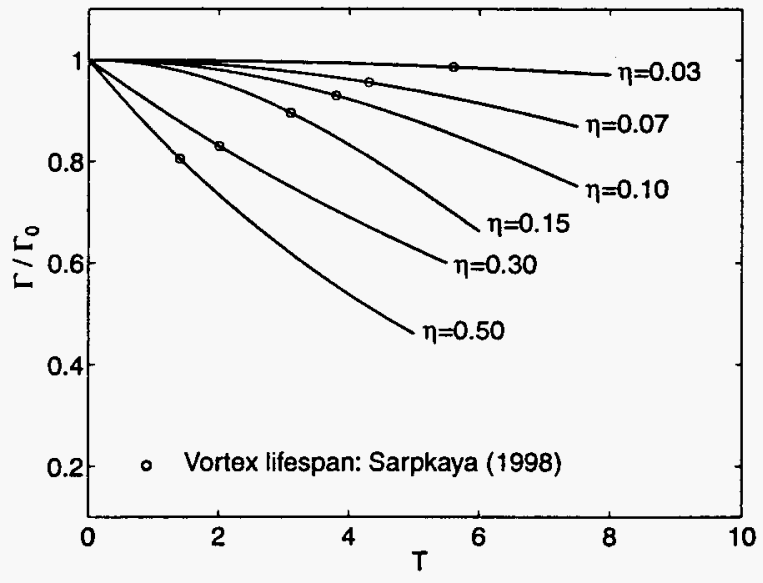

Figure 9: Model prediction with varying $\eta$ for the average circulation with vortex lifespan presented.

ticity is concentrated in two parallel lines, will follow the relation of $H=T$ in an inviscid fluid. In the numerical simulation, however, descent rate of a pair of the vortices can deviate from $H / T=1$ due to the influence of image vortices implicit upon the assumption of periodic boundary conditions and the use of a vortex model different from ideal line vortices. From a two-dimensional laminar simulation with the same domain size as the crossplane size of the previous three-dimensional simulation and with a very small constant viscosity $\left(R_{e} \equiv \Gamma / \nu \sim 10^{7}\right)$, the descent rate intrinsic in the present domain size and vortex model appears to be $H / T=0.98$, slightly less than ideal one.

In order to develop a model for vortex descent due to ambient turbulence, we first assume that the vortex descent rate is proportional to the circulation of the accompanying vortex at $r=b_{0}$, i.e.,

$$
\frac{d h}{d t} \sim \frac{\Gamma_{1}}{2 \pi b_{0}},
$$

where $\Gamma_{1}$ is the circulation at $r=b_{0}$. In the dimensionless form

$$
\frac{d H}{d T} \sim \frac{\Gamma_{1}}{\Gamma_{\infty}} .
$$

As discussed in the previous section, the circulation at $r=b_{0}$ is expected to decay as a Gaussian function. Thus, substituting $E q .(16)^{* *}$ into $E q .(18)$, we obtain

$$
\frac{d H}{d T} \sim e^{-\left(d_{1} \eta T\right)^{2}},
$$

\footnotetext{
${ }^{* *}$ At $r=b_{0}, E q .(16)$ can be written as $\Gamma=\Gamma_{0} e^{-c_{2} \eta^{2} T^{2}}$.
} 
where $d_{1}$ is an empirical coefficient proportional to $c_{2}$ and an approximation $\Gamma_{1}(T=0) \approx \Gamma_{\infty}$ is used. After integration, we finally obtain

$$
H=\frac{0.87}{d_{1} \eta} \operatorname{erf}\left(d_{1} \eta T\right),
$$

where erf denotes an error function and the constant 0.87 is an optimal value determined from our LES results.

The above model for vortex descent has been applied to our LES results for weak and moderate turbulence. As shown in Figs. 10a-d, the model predictions agree well with the LES data, although the coefficient $d_{1}$ appears to fluctuate with varying $\eta$. For strong turbulence, however, an optimal value for $d_{1}$ has not been found. This may be due to the dominance of the exponential decay of the circulation over radial distances less than about $0.6 b_{0}$, implying that the descent rate is not determined only by the circulations near $r=b_{0}$, but it is significantly influenced by those at small radial distances less than $b_{0}$. Nevertheless, for strong turbulence $E q .(20)$ can still be matched with the LES results by fixing $d_{1}$ and by changing the constant 0.87 to smaller values, as shown in Fig. 10e and f. For this case, therefore, the descent distance based on the LES results can be expressed by

$$
H=\frac{d_{2}}{0.28 \eta} \operatorname{erf}(0.28 \eta T),
$$

where $d_{2}$ is an empirical coefficient less than 0.87 .

The model coefficients $d_{1}$ and $d_{2}$ for more cases have been obtained and plotted together in Fig. 11 as done before for $c_{1}$ and $c_{2}$. As seen in Fig. 11, the coefficients fluctuate within only maximum $17 \%$ of their mean values of 0.71 for model $E$ and 0.84 for model $\mathrm{G}$, respectively.

Using the mean values of the coefficients, the model predictions for the vortex descent for various ambient turbulence levels are plotted in Fig. 12 again with the vortex lifespans obtained from Sarpkaya's ${ }^{10}$ model. Figure 12 shows clearly that the descent rate of the vortices decreases with increasing ambient turbulence due to the increasing rate of the circulation decay with increasing ambient turbulence. On the other hand, for weak turbulence, the descent distance appears to almost follow the ideal line $H=0.98 T$ before the vortex linking occurs.

\section{Comparison of observation data and model predictions}

In this section predictions of wake vortex decay and descent from the models proposed in the previous section are compared to observation from the Memphis Field Program. ${ }^{35}$ As part of the NASA sponsored field program, MIT Lincoln laboratory operated $10.6 \mu \mathrm{m} \mathrm{CO} \mathrm{O}_{2}$ continuous wave laser Doppler radar (Lidar) and measured the line-of-sight velocities of the vortices in a plane perpendicular to the fight path of approaching or departing aircraft. Circulation values were estimated from the measured line-of-sight velocity field. ${ }^{35}$

In this study, six cases (Table 2) are chosen based on atmospheric stability conditions and number of data points. Some of the above cases have been also analyzed by Sarpkaya ${ }^{10}$ for a different purpose. The proposed models only address the effect of ambient turbulence on vortex decay and did not include thermal stratification. The initial conditions for the proposed models used the representative vortex separation and far-field circulation in Table 2, as well as the TKE dissipation rate, $\epsilon$, measured at $40 m$ (Table 3). As seen from Table 3, the $\eta$ values calculated by the definition $(E q .(2))$ are within the range of those used in our previous LES.

Shown in Fig. 13 are the measured circulations averaged over a range of radial distances from $0.4 b_{0}$ to $0.6 b_{0}$ with model predictions based on $E q$.(13) and $E q .(14)$ using the mean coefficients. The initial average circulation $\left(\Gamma_{0}\right)$ is determined from the present vortex model $(E q .(8))$ with the theoretical circulation at $r \gg r_{c}\left(\Gamma_{\infty}\right)$ given in Table 2. The data for times less than $T=1$ has been discarded from the data set for all the cases analyzed, since the observed circulations in these times are subject to trigonometry errors (e.g., see Campbell et al. ${ }^{35}$ ).

As seen in Fig. 13, except for the cases in the stable stratification (M-1252 and M-1273), predictions by the proposed models appear to be in reasonable agreement with observations, although data scatter is large. Significant differences in the rate of decay for the weak turbulence cases of M-1252 and M-1273 between model predictions and observations may be caused by the interaction between vortex and stable stratification, which is not included in numerical simulations.

Also shown in Fig. 14 are the measured vortex descent with model predictions based on $E q$.(20) and $E q .(21)$ again using the mean coefficients. In the calculation, the starting altitude of the vortices has been inferred from the Lidar measurement as in Robins et al. ${ }^{36}$ since the vortices appears to be located above the expected position, i.e., the gen- 

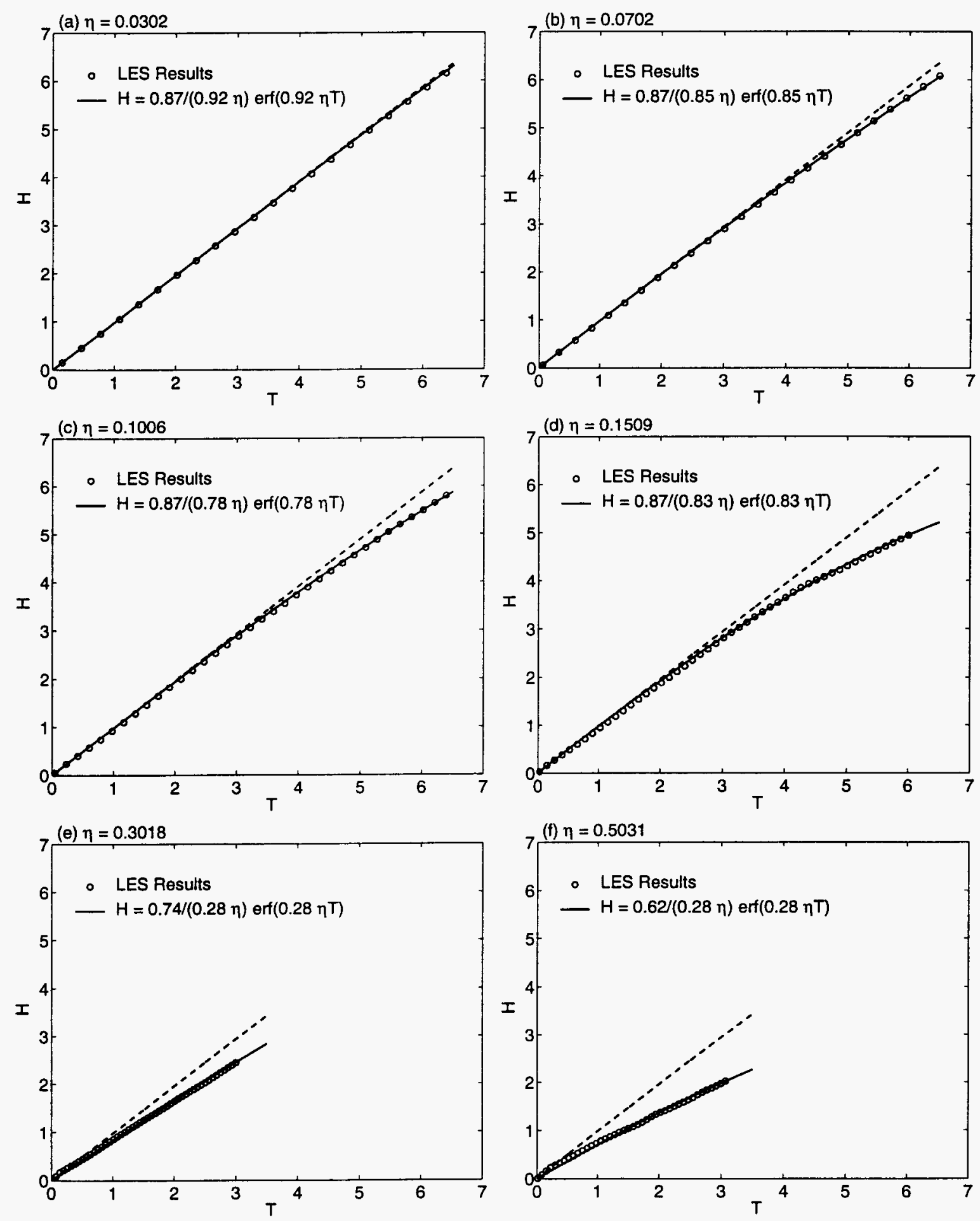

Figure 10: Evolution of the descent distance in three-dimensional simulations compared with the theoretical model. Dashed line denotes $H=0.98 T$. 


\begin{tabular}{|l|l|c|c|c|l|}
\hline \hline $\begin{array}{l}\text { Flight } \\
\text { number }\end{array}$ & $\begin{array}{l}\text { Aircraft } \\
\text { type }\end{array}$ & $\begin{array}{c}b_{0} \\
(m)\end{array}$ & $\begin{array}{c}\Gamma_{\infty} \\
\left(m^{2} s^{-1}\right)\end{array}$ & $\begin{array}{l}\text { Aircraft } \\
\text { altitude }(m)\end{array}$ & $\begin{array}{l}\text { Atmospheric stability } \\
\text { (observing time) }\end{array}$ \\
\hline $\mathrm{M}-1252$ & $\mathrm{~B}-757$ & 29.8 & 294 & 160.2 & stable (midnight) \\
\hline $\mathrm{M}-1273$ & $\mathrm{DC}-10$ & 39.6 & 400 & 149.9 & stable (midnight) \\
\hline $\mathrm{M}-1409$ & $\mathrm{~B}-727$ & 25.8 & 240 & 146.6 & neutral (evening) \\
\hline $\mathrm{M}-1569$ & $\mathrm{DC}-9$ & 22.4 & 215 & 127.5 & neutral (evening) \\
\hline $\mathrm{M}-1581$ & $\mathrm{~B}-757$ & 29.8 & 270 & 166.1 & unstable (noon) \\
\hline $\mathrm{M}-1584$ & $\mathrm{DC}-9$ & 22.4 & 199 & 125.7 & unstable (noon) \\
\hline \hline
\end{tabular}

Table 2: Initial parameters for wake vortices for the flights analyzed.

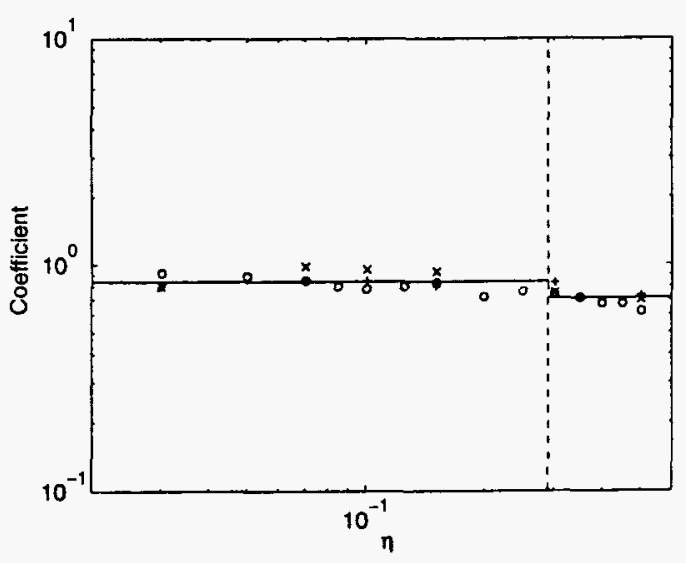

Figure 11: Same as Fig. 8 but for the vortex descent in $E q .(20)$ and $E q .(21)$.

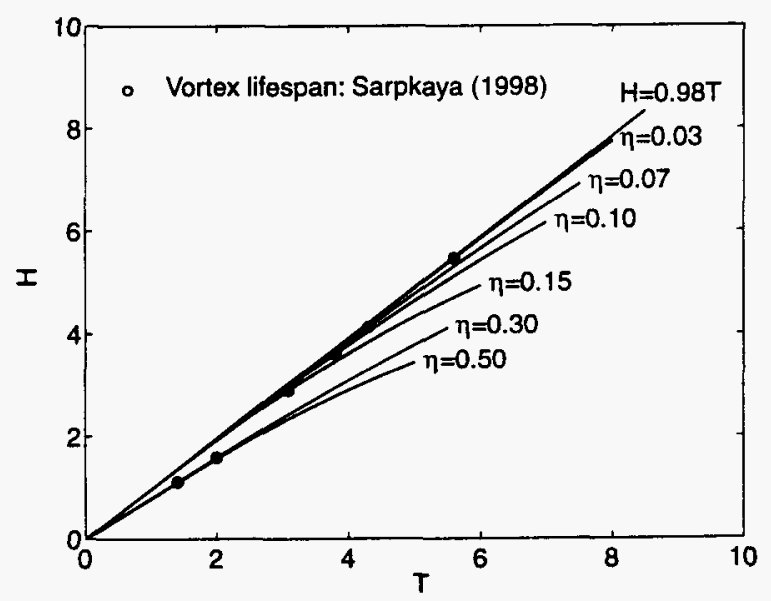

Figure 12: Same as in Fig. 9 but for the vortex descent.

\begin{tabular}{|l|c|c|}
\hline \hline $\begin{array}{l}\text { Flight } \\
\text { number }\end{array}$ & $\begin{array}{c}\epsilon \\
\left(\mathrm{m}^{2} \mathrm{~s}^{-3}\right)\end{array}$ & $\eta$ \\
\hline $\mathrm{M}-1252$ & $0.55 \times 10^{-5}$ & 0.03 \\
\hline $\mathrm{M}-1273$ & $0.20 \times 10^{-3}$ & 0.12 \\
\hline $\mathrm{M}-1409$ & $0.29 \times 10^{-2}$ & 0.28 \\
\hline $\mathrm{M}-1569$ & $0.79 \times 10^{-3}$ & 0.17 \\
\hline $\mathrm{M}-1581$ & $0.13 \times 10^{-1}$ & 0.51 \\
\hline $\mathrm{M}-1584$ & $0.10 \times 10^{-1}$ & 0.43 \\
\hline
\end{tabular}

Table 3: Turbulence energy dissipation rate measured at elevation of $40 \mathrm{~m}$ and corresponding dimensionless turbulence intensity.

erating aircraft height in Table 2. Consistent with the large underestimation for the circulation decay, the model predictions in the cases of M-1252 and M-1273 significantly overestimate the observed vortex descent especially at later time period. Except for the case of M-1409 (Fig. 14c), the model predictions for the other cases appear to be in reasonable agreement with observations. The underprediction in the case of M-1409 could be caused by other factors such as wind shear or large-scale atmospheric vertical motion.

\section{Summary and conclusions}

This study represents a first step at understanding how wake vortices behave in the atmosphere by isolating the effects of ambient turbulence on vortex decay. LES simulations are conducted by first growing a field of ambient turbulence which is nearly isotropic and spatially homogeneous. A vortex pair, representative of aircraft trailing vortices, is introduced once the turbulence field reaches a steady state. Results show that the decay rate of the vortex circulation increases clearly with increasing levels of ambient turbulence. In these simulations Crow link- 

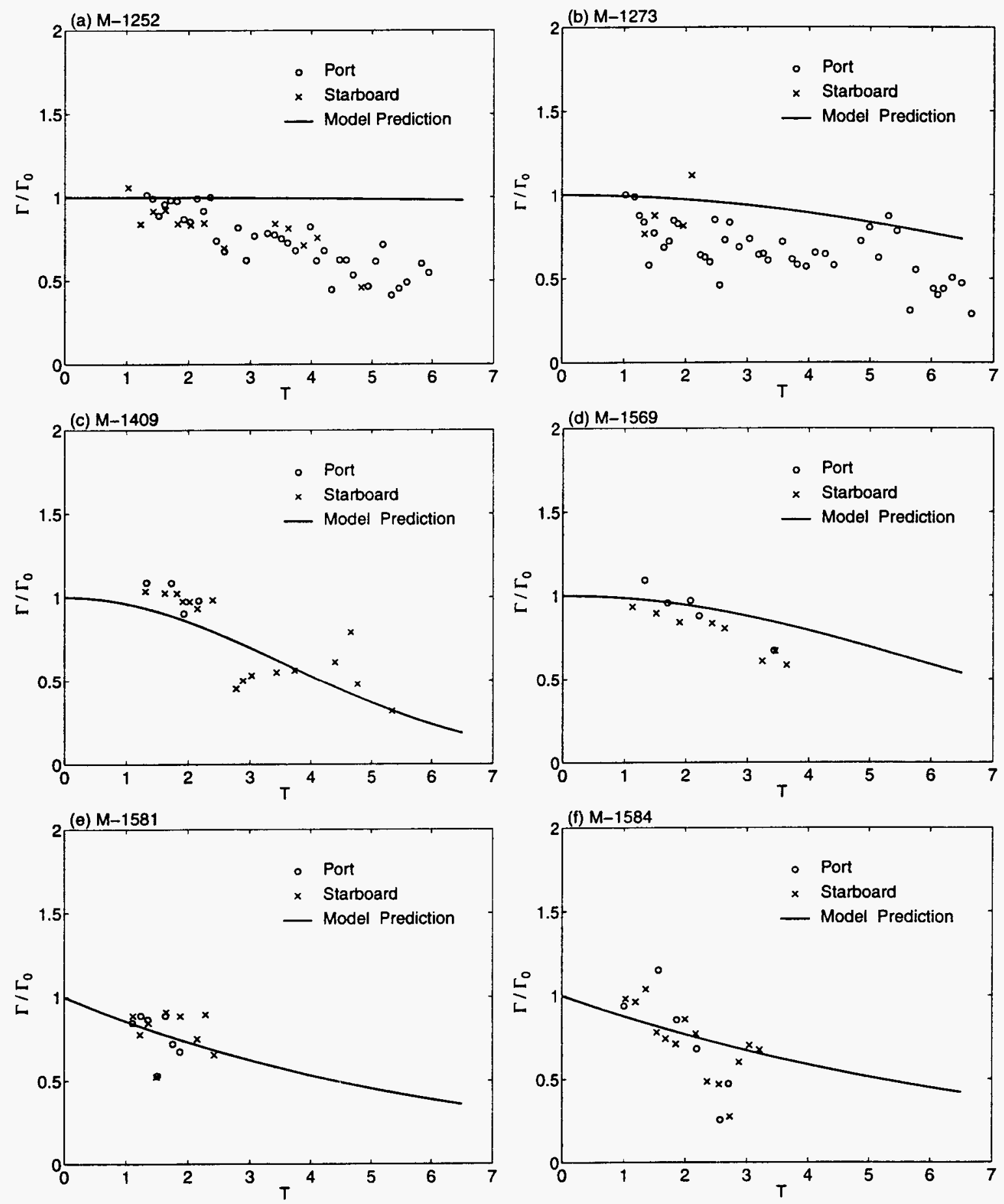

Figure 13: Comparison of field observations and model predictions for the average circulation; (a) and (b): stable environment; (c) and (d): neutral environment; (e) and (f): unstable environment. 

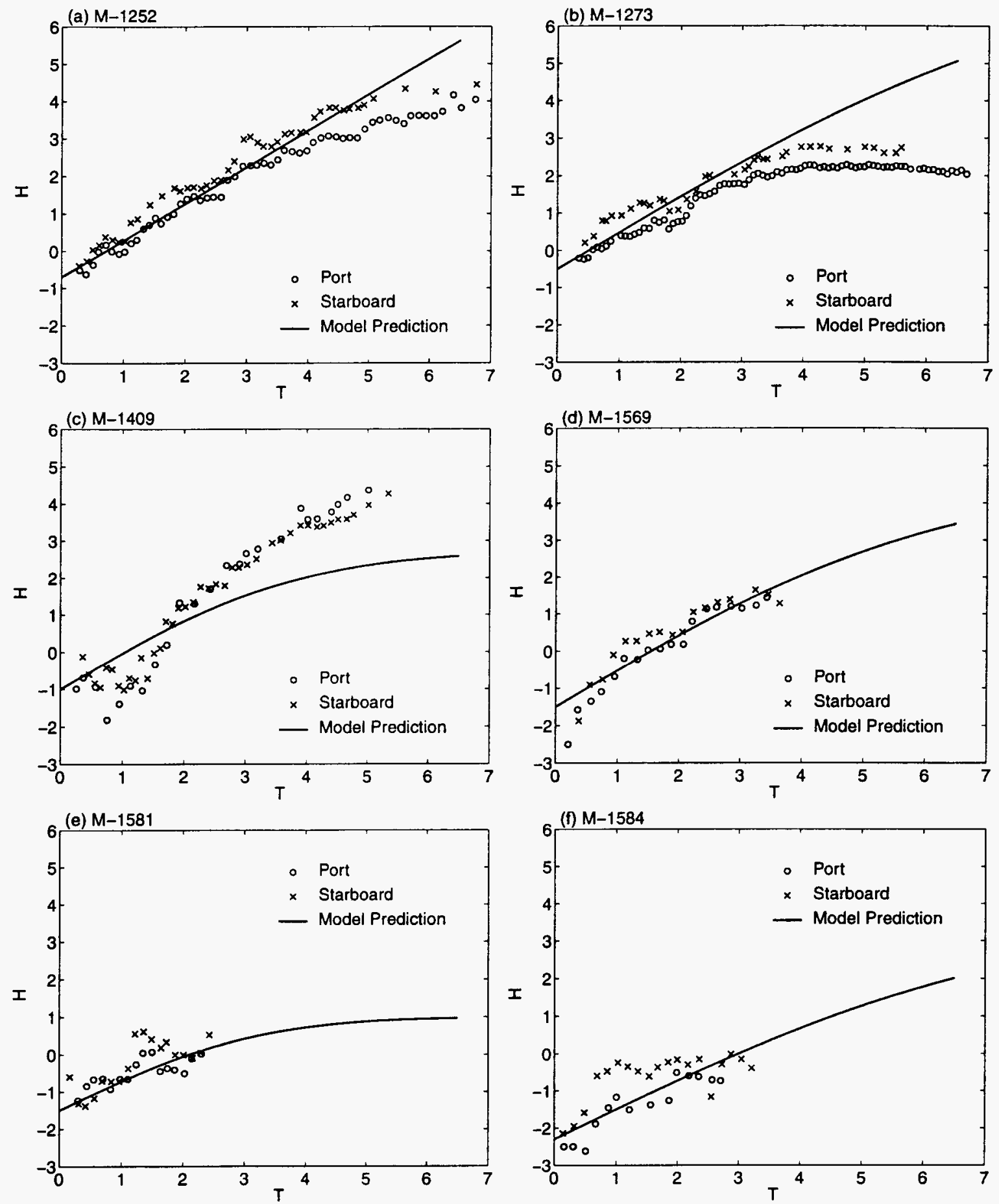

Figure 14: Same as Fig. 13 but for the vortex descent. 
ing and its subsequent effect on vortex decay are suppressed by the choice of domain size.

Based on the LES results, simple decay models are proposed as functions of dimensionless ambient turbulence intensity $(\eta)$ and dimensionless time $(T)$ for the circulation averaged over radial distances from $0.4 b_{0}$ to $0.6 b_{0}$. With good agreement with the LES data, a Gaussian type of vortex decay model is applicable for weak and moderate turbulence, while an exponential type of vortex decay model can be applied for strong turbulence. The LES simulations and the fitted models show that the turbulence dissipation rate characterizes the level of ambient turbulence responsible for vortex decay. A model for the vortex descent based on above vortex decay model is also proposed as functions of $\eta$ and $T$ and can be represented by an error function. The proposed models for the vortex decay and descent are applied to available field data obtained from the Memphis airport. In a turbulent atmosphere with either neutral or unstable stratification, the model predictions appear to be in reasonable agreement with the observation data. In a stably-stratified atmosphere with low turbulence, the models largely underestimate the observed circulation decay with consistent overestimation of the observed vortex descent. The lack of agreement in the latter is attributed to the effects of stratification on vortex decay.

\section{Acknowledgments}

This work was supported by NASA's Terminal Area Productivity program under Contract \# NAS 1-18925 (Cooperative Agreement \# NCC-1-188). Numerical simulations were carried out on NASA's Cray C90 and J90 and North Carolina Supercomputing Center's Cray T916. Discussions with Drs. C.-T Kao and S. Shen at North Carolina State University are highly appreciated.

\section{References}

1. Hinton, D. A., "Aircraft Vortex Spacing System (AVOSS) Conceptual Design," NASA TM-110184, August 1995.

2. Perry, R. B., Hinton, D. A., and Stuever, R. A., "NASA Wake Vortex Research for Aircraft Spacing," 35th Aerospace Sciences Meeting \& Exhibit, Reno, NV, AIAA Paper No. 97-0057, January 1997.
3. Proctor, F. H., "The NASA-Langley Wake Vortex Modeling Effort in Support of an Operational Aircraft Spacing System," 36th Aerospace Sciences Meeting \& Exhibit, Reno, NV, AIAA Paper No. 980589, January 1998.

4. Spalart, P. R., "Wake Vortex Physics: The Great Controversies," NASA First Wake Vortex Dynamic Spacing Workshop, Proceedings, Hampton, VA, NASA CP-97-206235, May 1997, pp. 3334.

5. Crow, S. C., "Stability Theory for a Pair of Trailing Vortices," AIAA Journal, Vol. 8, 1970, pp. 2172-2179.

6. Spalart, P. R., "Airplane Trailing Vortices," $A n$ nual Reviews of Fluid Mechanics, Vol. 30, 1998, pp. 1-35.

7. Donaldson, C. duP. and Bilanin, A. J., "Vortex Wakes of Conventional Aircraft," AGARDograph No. 204, May 1975.

8. Greene, G. C., "An Approximate Model of Vortex Decay in the Atmosphere," Journal of Aircraft, Vol. 23, 1986, pp. 566-573.

9. Bilanin, A. J., Teske, M. E., and Hirsch, J. E., "Neutral Atmospheric Effects on the Dissipation of Aircraft Vortex Wakes," AIAA Journal, Vol. 16, 1978, pp. 956-961.

10. Sarpkaya, T., "Decay of Wake Vortices of Large Aircraft," AIAA Journal, Vol. 36, 1998, pp. 16711679.

11. Han, J., Lin, Y. -L., Schowalter, D. G., Arya, S. P., and Proctor, F. H., "Large-Eddy Simulation of Aircraft Wake Vortices: Atmospheric Turbulence Effects," 12th Symposium on Boundary Layers and Turbulence, Vancouver, Canada, July-August 1997, pp. 237-238.

12. Han, J., Lin, Y. -L., Arya, S. P., and Kao, C., "Large-Eddy Simulation of Aircraft Wake Vortices: Atmospheric Turbulence Effects," NASA First Wake Vortex Dynamic Spacing Workshop, Proceedings, Hampton, VA, NASA CP-97-206235, May 1997, pp. 131-144.

13. Sarpkaya, T. and Daly, J. J., "Effect of Am- 
bient Turbulence on Trailing Vortices," Journal of Aircraft, Vol. 24, 1987, pp. 399-404.

14. Sarpkaya, T., "Trailing Vortices in Homogeneous and Density-Stratified Media," Journal of Fluid Mechanics, Vol. 136, 1983, pp. 85-109.

15. Hallock, J. N. and Burnham, D. C., "Decay Characteristics of Wake Vortices from Jet Transport Aircraft," 35th Aerospace Sciences Meeting \& Exhibit, Reno, NV, AIAA Paper No. 97-0060, January 1997.

16. Tombach, I., "Observations of Atmospheric Effects on Vortex Wake Behavior," Journal of Aircraft, Vol. 10, 1973, pp. 641-647.

17. Spalart, P. R. and Wray, A. A., "Initiation of the Crow Instability by Atmospheric Turbulence," 78th AGARD-FDP Symposium on the Characterization and Modification of Wakes from Lifting Vehicles in Fluids, Trondheim, Norway, 20-23 May 1996.

18. Han, J., Lin, Y. -L., Schowalter, D. G., Arya, S. P., and Proctor, F. H., "Large Eddy Simulation of Aircraft Wake Vortices in a Homogeneous Atmospheric Turbulence: The Crow Instability," Accepted for publication in AIAA Journal, 1998.

19. Kolmogorov, A. N., "The Local Structure of Turbulence in Incompressible Viscous Fluid for Very Large Reynolds Number," Dokl. Akad. Nauk SSSR, Vol. 30, 1941, pp. 9-13.

20. Crow, S. C. and Bate, E. R., "Lifespan of Trailing Vortices on a Turbulent Atmosphere," Journal of Aircraft, Vol. 7, 1976, pp. 476-482.

21. Liu, H. -T., "Effects of Ambient Turbulence on the Decay of a Trailing Vortex Wake," Journal of Aircraft, Vol. 29, 1992, pp. 255-263.

22. Proctor, F. H., "The Terminal Area Simulations System, Volume 1: Theoretical Formulation," NASA Contractor Report 4046, DOT/FAA/PM-86/ $50,1,1987$.

23. Proctor, F. H., "Numerical Simulation of Wake Vortices Measured During the Idaho Fall and Memphis Field Programs," 14th AIAA Applied Aerodynamics Conference, Proceedings, Part-2, New Orleans, LA, AIAA Paper No. 96-2496, June 1996. pp. $943-960$.

24. Proctor, F. H., Hinton, D. A., Han, J., Schowalter, D. G., and Lin, Y. -L., "Two-Dimensional Wake Vortex Simulations in the Atmosphere: Preliminary Sensitivity Studies," 35th Aerospace Sciences Meeting \& Exhibit, Reno, NV, AIAA Paper No. 97-0056, January 1997.

25. Schowalter, D. G., Decroix, D. S., Switzer, G. F., Lin, Y. -L., and Arya, S. P., "Toward ThreeDimensional Modeling of a Wake Vortex Pair in the Turbulent Boundary Layer," 35th Aerospace Sciences Meeting \& Exhibit, Reno, NV, AIAA Paper No. 97-0058, January 1997.

26. Lord Rayleigh, "On the Dynamics of Revolving Fluids," Proc. Roy. Soc., A93, 1916, pp. 148.

27. Smagorinsky, J., "General Circulation Experiments with the Primitive Equations: I. The Basic Experiments," Monthly Weather Review, Vol. 91, 1963, pp. 99-164.

28. Proctor, F. H., will appear in NASA Technical Note.

29. Lamb, H., Hydrodynamics, 6th Ed., Cambridge University Press, 1932, 738 pp.

30. Corjon, A., Risso, F., Stoessel, A., and Poinsot, T., "Three-Dimensional Direct Numerical Simulations of Wake Vortices: Atmospheric Turbulence Effects and Rebound with Crosswind," 78th AGARDFDP Symposium on The Characterization and Modification of Wakes from Lifting Vehicles in Fluids, Trondheim, Norway, May 1996.

31. Corjon, A. and Darracq, D., "Three-Dimensional Large Eddy Simulation of Wake Vortices. Comparison with Field Measurements," 15th AIAA Applied Aerodynamics Conference, Atlanta, GA, AIAA Paper No. 97-2309, June 1997. 11pp.

32. Burnham, D. C. and Hallock, J. N., "Chicago Monostatic Acoustic Vortex Sensing System," Report No. DOT-TSC-FAA-79-103IV, July 1982, 206 pp.

33. Hinton, D. A. and Tatnall, C. R., "A Candidate Wake Vortex Strength Definition for Application to the NASA Aircraft Vortex Spacing System 
(AVOSS)," NASA TM-110343, September 1997, 35

pp.

34. Tatnall, C. R., "An Investigation of Candidate Sensor-Observable Wake Vortex Strength Parameters for the NASA Aircraft Vortex Spacing System (AVOSS)," NASA/CR-1998-206933, March 1998, 30 pp.

35. Campbell, S. D., Dasey, T. J., Freehart, R. E., Heinrichs, R. M., Matthews, M. P., Perras, G. H., and Rowe, G. S., "Wake Vortex Field Measurement Program at Memphis, TN Data Guide," Project Report: $N A S A / L-2$, January 1997.

36. Robins, R. E., Delisi, D. P., and Greene, G. C., "Development and Validation of a Wake Vortex Predictor Algorithm," 36th Aerospace Sciences Meeting \& Exhibit, Reno, NV, AIAA Paper No. 98-0665, January 1998.

\section{Appendix A}

According to Donaldson and Bilanin, ${ }^{7}$ hereafter referred as DB, for the neutrally stratified and high Reynolds number flows, the equation that governs the rate of change for mean axial vorticity $\bar{\zeta}$ due to turbulent motion is given by

$$
\frac{d \bar{\zeta}}{d t}=-\frac{\partial}{\partial x_{j}} \overline{u_{j}^{\prime} \zeta^{\prime}} .
$$

Integration of $E q .(A 1)$ over region defined by the radial distance $r$ yields

$$
\iint \frac{d \bar{\zeta}}{d t} d A=-\iint \frac{\partial}{\partial x_{j}} \overline{u_{j}^{\prime} \zeta^{\prime}} d A .
$$

The area integral on the right-hand side of $E q .(A 2)$ can be transformed into a line integral taken around the perimeter of the area using the Stoke's theorem, i.e.,

$$
\frac{d \Gamma}{d t}=-\oint \overline{u_{n}^{\prime} \zeta^{\prime}} d s,
$$

where $u_{n}^{\prime}$ is the fluctuation of velocity normal to the surface. The line integral leads to

$$
\frac{d \Gamma}{d t}=-2 \pi r\left\langle\overline{u_{n}^{\prime} \zeta^{\prime}}\right\rangle
$$

where \langle\rangle denotes an average around the perimeter with the radial distance $r$. Under down-gradient transport assumption,

$$
\left\langle\overline{u_{n}^{\prime} \zeta^{\prime}}\right\rangle=-\nu_{e} \frac{\partial \bar{\zeta}}{\partial r},
$$

where $\nu_{e}$ is an effective eddy viscosity. The radial gradient of mean axial vorticity may be approximated by

$$
\frac{\partial \bar{\zeta}}{\partial r} \sim-\frac{\bar{\zeta}}{r}
$$

and

$$
\bar{\zeta} \sim \frac{\Gamma}{r^{2}},
$$

so that

$$
\frac{\partial \bar{\zeta}}{\partial r} \sim-\frac{\Gamma}{r^{3}} .
$$

Note that $\partial \bar{\zeta} / \partial r$ is assumed to be proportional to the difference of mean axial vorticity at the vortex center and that at radial distance $r$. Thus, $E q$. $(A 4)$ can be expressed as

$$
\frac{d \Gamma}{d t} \sim-\frac{\nu_{e} \Gamma}{r^{2}} .
$$

Above derivations are almost the same as DB's except for the fact that the dependency of circulation decay on the radial distance $r$ is considered in the present study, while $b_{0}$ is used instead of $r$ in DB's study, since DB consider the decay rate of the circulation over an oval within which a pair of vortices resides.

From an equilibrium second-order closure theory, DB obtain the relation

$$
\nu_{e}=0.26 \Lambda q,
$$

where $\Lambda$ is a measure of the integral length scale of turbulence. Assuming that

$$
\Lambda \simeq \frac{b_{0}}{8},
$$

DB then obtain $E q .(1)$. However, their approximation of $E q .(A 11)$ is not physically correct, since $b_{0}$ has no relation with background atmospheric turbulence whose length scale varies significantly over day and night. As already discussed in the introduction, in this study we use $\epsilon$, rather than $q$ and $\Lambda$, as the ambient turbulence parameter relevant to circulation decay.

For strong turbulence but at smaller radial distances where stronger radial gradient of mean axial vorticity may exist, the parameterization of $E q .(A 5)$ would be acceptable. The effective eddy viscosity in 
this case can be expressed in terms of effective turbulence velocity and length scales, i.e.,

$$
\nu_{e} \sim\left(\epsilon b_{0}\right)^{1 / 3} b_{0} .
$$

Note that $\left(\epsilon b_{0}\right)^{1 / 3}$ is a characteristic turbulence velocity scale at the scale of the vortex separation distance $b_{0}$. Substituting $E q .(A 12)$ into $E q .(A 9)$, we obtain

$$
\frac{d \Gamma}{d t} \sim-\frac{\left(\epsilon b_{0}\right)^{1 / 3} b_{0}}{r^{2}} \Gamma .
$$

Thus, the decay rate of the circulation depends on both the turbulence energy dissipation rate $\epsilon$ and radial distance $r$, unlike DB's model (Eq.(1)). Equation $(A 13)$ can be nondimensionalized as

$$
\frac{d \Gamma}{d T} \sim-\frac{\eta}{R^{2}} \Gamma
$$

For weak and moderate turbulence or at larger radial distances for strong turbulence where the radial gradient of mean axial vorticity may be weak, the parameterization of $E q .(A 5)$ would not be acceptable. It is anticipated that the radial turbulent vorticity flux $\overline{u_{n}^{\prime} \zeta^{\prime}}$ in this case will be mostly influenced by turbulent diffusion of vorticity from the core region rather than by local diffusion like in $E q .(A 5)$. This implies that $\overline{u_{n}^{\prime} \zeta^{\prime}}$ would be time dependent. We can hypothesize that the change rate of $\overline{u_{n}^{\prime} \zeta^{\prime}}$ in this case depends on turbulence velocity and mean axial vorticity gradient from vortex center to given $r$, i.e.,

$$
\frac{\partial\left(\overline{u_{n}^{\prime} \zeta^{\prime}}\right)}{\partial t}=f\left(\left(\epsilon b_{0}\right)^{1 / 3}, \frac{\Gamma}{r^{3}}\right) .
$$

From dimensional arguments,

$$
\frac{\partial\left(\overline{u_{n}^{\prime} \zeta^{\prime}}\right)}{\partial t} \sim-\frac{\left(\epsilon b_{0}\right)^{2 / 3} \Gamma}{r^{3}} .
$$

Integration of $E q .(A 16)$ with respect to time leads to

$$
\overline{u_{n}^{\prime} \zeta^{\prime}} \sim-\frac{\left(\epsilon b_{0}\right)^{2 / 3} t \Gamma}{r^{3}}
$$

where the initial value of $\overline{u_{n}^{\prime} \zeta^{\prime}}$ is assumed to be zero. Substituting $E q .(A 17)$ into $E q .(A 4)$, we obtain

$$
\frac{d \Gamma}{d t} \sim-\frac{\left(\epsilon b_{0}\right)^{2 / 3} t}{r^{2}} \Gamma .
$$

The above equation can be nondimensionalized as

$$
\frac{d \Gamma}{d T} \sim-\frac{\eta^{2} T}{R^{2}} \Gamma .
$$

American Institute of Aeronautics and Astronautics 